\title{
El auge del aprendizaje universitario ubicuo. Uso de las tabletas en la apropiación del conocimiento
}

\section{The boom in ubiquitous university learning. Use of tablets in the appropriation of knowledge}

\author{
BLANCA INÉS ESPINEL*1 \\ bespinel@tecnologicocomfenalco.edu.co \\ María luisa Sevillano García** \\ mlsevillano@edu.uned.es \\ Iván Javier Monterrosa Castro* \\ imonterrosa@tecnologicocomfenalco.edu.co \\ Carolina Pascual Moscoso** \\ Cpascual90@edu.uned.es \\ * Fundación Universitaria Tecnológico Comfenalco - Cartagena, Colombia \\ ** Universidad Nacional de Educación a Distancia, España
}

\section{Resumen:}

El artículo se propone abordar las nuevas formas de aprendizaje ubicuo con las tabletas en el ámbito universitario actual. Vincular las tabletas a la universidad con la comunidad educativa, es una de las exigencias metodológicas activas que el alumnado requiere para explorar algunos de los cambios que los nuevos contextos en la formación del estudiante universitario, así como la forma en que éstas impactan en el pensamiento y el aprendizaje, encaminadas a partir de la respuesta que brinda a las demandas y necesidades que se presentan en el ámbito académico. A través del diseño de investigación descriptivo, con un enfoque mixto, se analiza una muestra de 213 estudiantes de dos universidades, integrando las técnicas cualitativas (directivos) y cuantitativas. Para la

\begin{abstract}
:
This article aims to address the new forms of ubiquitous learning with tablets in current university environments. Bringing tablets into the university educational community is one of the methodological demands that students require in order to explore some of the changes in the new contexts which define university students' education. Besides, it is also necessary to shed light on the impact of tablets on the thinking and learning processes which academic contexts aim to activate in students. Through a descriptive research design, with a mixed-method approach, a sample of 210 students from two universities is analyzed, integrating qualitative (managing staff) and quantitative techniques. To validate the survey, the technique of experts' opinions was applied - the experts were
\end{abstract}

1 Dirección para correspondencia (correspondence address):

Blanca Inés Espinel. Fundación Universitaria Tecnológico Comfenalco - Cartagena. Cra. 44 \#30a-91, Cartagena, Provincia de Cartagena, Bolívar (Colombia). 
El auge del aprendizaje universitario ubicuo. Uso de las tabletas en la apropiación del conocimiento

Blanca Inés Espinel, María luisa Sevillano García, Iván Javier Monterrosa Castro y

Carolina Pascual Moscoso

validación de la encuesta, se utilizó la técnica de expertos, seleccionados mediante el procedimiento de "Coeficiente K». El análisis del cuestionario se hizo a través de la prueba de Cronbach, siendo significativa con 0,897 de grado de fiabilidad. Los resultados señalaron que existe un desconocimiento sobre su función pedagógica, poca valoración y preparación para emplearla en el aprendizaje y para cambiar las prácticas formativas tradicionales. Además, una oportunidad de aprovechar estos dispositivos es poder articular conocimientos académicos, científicos y tecnológicos, con las diferentes realidades que germinan en el contexto universitario. Se concluye que el uso de las tabletas es un campo de investigación y práctica educativa en auge por las tecnologías, por lo que se hace necesario conocer su potencial en la apropiación del conocimiento en la educación y el aprendizaje significativo.

\section{Palabras clave:}

Tableta; aprendizaje ubicuo; formación; enseñanza; ambientes virtuales. selected through the "Coefficient K" procedure. The analysis of the questionnaire was done through a Cronbach test and the degree of reliability obtained was significant $(0,897)$. The results reveal a certain resistance to change traditional teacher training practices, a poor perception of the educational potential of tablets and a lack of knowledge about their pedagogical role. A clear possibility to take advantage of these devices consists of articulating academic, scientific and technological knowledge based on the different realities which emerge from university contexts. The present article concludes by stating that the use of tablets is a research strand which is booming due to new technologies and, therefore, it seems necessary to raise awareness of its great potential when it comes to the articulation of knowledge and the implementation of meaningful learning.

\section{Key words:}

Tablet; ubiquitous learning; education; teaching; virtual environments.

\section{Résumé:}

L'article vise à aborder les nouvelles formes d'apprentissage omniprésent avec des tablettes dans l'environnement universitaire actuel. Relier les tablettes à l'université avec la communauté éducative est l'une des exigences méthodologiques actives dont l'étudiant a besoin pour explorer certains des changements que les nouveaux contextes dans la formation de l'étudiant universitaire, ainsi que la façon dont ceux-ci influent sur la pensée et l'apprentissage, visant à répondre aux demandes et aux besoins qui se posent dans le domaine académique. À travers la conception de la recherche descriptive, avec une approche mixte, un échantillon de 213 étudiants de deux universités est analysé, intégrant des techniques qualitatives (managériales) et quantitatives. Pour la validation de l'enquête, nous avons utilisé la technique des experts, sélectionnés à travers la procédure "Coefficient K". L'analyse du questionnaire a été faite à travers le test de Cronbach, étant significatif avec un degré de fiabilité de 0,897. Les résultats ont indiqué qu'il y a un manque de connaissance sur sa fonction pédagogique, peu d'évaluation et de préparation pour l'utiliser dans l'apprentissage et pour changer les pratiques de formation traditionnelles. De plus, une opportunité de tirer parti de ces dispositifs est de pouvoir articuler les connaissances académiques, scientifiques et technologiques, avec les différentes réalités qui germent dans le contexte universitaire. II est conclu que l'utilisation des tablettes est un domaine de recherche et de pratique éducative en plein essor sur les technologies, il est donc nécessaire de connaître leur potentiel dans l'appropriation des connaissances dans l'éducation et l'apprentissage significatif.

\section{Mots clés:}

Tablette; apprentissage omniprésent; éducation; enseignement; environnements virtuels.

Fecha de recepción: 13-4-2018

Fecha de aceptación: 1-10-2018 
Blanca Inés Espinel, María luisa Sevillano García, Iván Javier Monterrosa Castro y

\section{Introducción}

En este texto se muestran los resultados del estudio cuyo objetivo era identificar usos y frecuencias de las tablas agrupadas por participantes y explorar las competencias que los estudiantes investigados manifiestan precisar para un buen uso de las mismas.

El texto está estructurado por seis sesiones: Inicia con la descripción y justificación del estudio. Segunda se demuestran los fundamentos teóricos que acompañan el estudio. En tercera se agrupa los aspectos metodológicos fundados en un diseño de investigación descriptivo de tipo mixto, con una mezcla de enfoques cuantitativos y cualitativos que conllevan identificar y expresar las ideas y/o apreciaciones de cada uno de los participantes con respecto al uso y frecuencia que emplean las tablas con fines pedagógicos. En la cuarta se presentan efectos alcanzados a través de la encuesta como en los grupos de discusión y de las entrevistas formales, integrando las relaciones entre las puntuaciones obtenidas por los participantes y los distintos factores objeto de estudio en la encuesta, así como el análisis de contenido referente a las entrevistas y grupos de discusión. El quinto se dedica a las conclusiones.

\section{Nuevas formas de aprendizaje universitario}

Hay acciones como la formación, la investigación, el asesoramiento que pone en valor el conocimiento inherente a la tecnología transferida (San Martín, Martín \& Ramírez, 2016, p. 353). Los estudiantes universitarios en Cartagena, según los estudios cimentados en las nuevas teorías emergentes de la comunidad científica, indican que, junto a múltiples disfunciones, hoy se tiene el reto y la responsabilidad de convertirlos en aliados de la docencia mediante una acertada programación didáctica (Sevillano-García \& Vázquez-Cano, 2015, p. 116).

Trigueros (2017) señala la importancia que tiene formar: "Una línea de investigación interesante sería analizar la posición de este alumnado frente a la explosión de aplicaciones aportadas por los dispositivos móviles de Internet, con grandes potencialidades de uso en su vida diaria"

Ibáñez (2004) asegura: "Las instituciones de educación superior deben flexibilizarse y desarrollar vías de integración de las tecnologías de la información y la comunicación en los procesos de formación» (p. 16); 
El auge del aprendizaje universitario ubicuo. Uso de las tabletas en la apropiación del conocimiento

Blanca Inés Espinel, María luisa Sevillano García, Iván Javier Monterrosa Castro y Carolina Pascual Moscoso

es decir, adaptarse a las necesidades de la sociedad actual. Ya en Colombia, específicamente en la región del Caribe (Said-Hung, 2015) han tenido un proyecto muy interesante sobre el fortalecimiento pedagógico en las universidades a través de las TIC. Se constata que es necesario el aprovechamiento de los recursos pedagógicos dispuestos en internet, así como la necesidad de garantizar la mayor promoción y generación de profesores eficaces en los escenarios de enseñanza superior a nivel de apropiación tecnológica. También en Bogotá (Colombia se realizó una investigación muy interesante sobre la brecha digital universitaria y la apropiación de las TIC en estudiantes de Educación Superior (Berrio Zapata, 2014) Según este estudio, los estudiantes universitarios usan las TIC, pero dada su formación y educación no se apropian de sus posibilidades productivas más allá de los usos básicos o recreativos. La apropiación tecnológica es un fenómeno individual pero también colectivo que incluye factores políticos, económicos y culturales que deben ser analizados en su conjunto.

Martínez \& Murillo (2016) advierten la necesidad de: "buscar aportar algunas ideas que potencien su labor, que contribuyan a una reflexión informada que desemboque en una mejora de los procesos de enseñanza y aprendizaje. Si y solo si confiamos en la competencia y profesionalidad de los y las docentes iberoamericanos, solo si potenciamos su rol de intelectuales críticos y transformadores, estaremos contribuyendo a un cambio en la educación", razón del porqué la tableta sería una herramienta esencial para lograr impactar la educación de calidad.

Cossio \& Hernández (2016) aseguran la necesidad de que los docente adquieran competencia para la enseñanza: “... la mayoría de sus discursos carecen de una adecuada comprensión de las nuevas propuestas educativas, lo que provoca que los profesores no cuenten con los suficientes elementos para resignificar sus representaciones del aprendizaje y por lo tanto sus prácticas de enseñanza se muestran alejadas de una postura predominantemente constructiva" en este caso incorporar las tabletas a la práctica sería un potencial.

Durán, Gutiérrez \& Prendes (2016). Exponen la necesidad para la mejora de la docencia en la educación superior, formación en competencia TIC de este colectivo. "Dentro del contexto universitario, donde cada vez se le da más prioridad a la adecuada formación en competencias digitales".

La tableta (del inglés tablet o tablet computer) es un tipo de compu- 
Blanca Inés Espinel, María luisa Sevillano García, Iván Javier Monterrosa Castro y

tadora portátil con la que se interactúa principalmente con los dedos o un bolígrafo stylus (pasiva o activa), sin necesidad de teclado físico ni ratón (mouse).

En las discusiones sobre la reforma educativa del acceso a la función docente, se valora la posibilidad de incluir entre las temáticas de examen obligatorio donde los aspirantes deben acreditar una suficiente competencia digital apropiada para la función docente (San Martín, 2014, p. 14).

Este artículo se centra en un dispositivo: la tableta, que para su uso también es necesaria la competencia digital. Según, Katja Friedrich (2012), el concepto móvil, dispositivo móvil, incluso unido a ubicuo, puede aplicarse hasta a un libro en papel, una revista; no obstante, se estima que cuando se habla de tableta como dispositivo móvil facilitador de un aprendizaje ubicuo, se está operando con un elemento sustancial que enriquece la movilidad-ubicuidad, que es la universalidad en cuanto a contenidos posibles y la actualización permanente, frente a la esteticidad y cierre de los otros formatos.

Mas-Torelló \& Olmos-Rueda (2016), explican la necesidad de formación continua que debe tener el docente para facilitar la actuación en aula: "necesario el diseño de un plan de formación continua igualmente contextualizado y específico, para que el profesorado universitario pueda mejorar su práctica profesional" Por lo anteriormente indicado aprender a utilizar la tableta pedagógicamente sería un dispositivo ideal en su práctica.

La potencialidad de los dispositivos móviles y ubicuos constituye un factor importante en la vida de los jóvenes, como señala Hagen (2013); son estos nuevos elementos los que aportan dimensiones sustanciales y significativas a los procesos de aprendizaje y enseñanza. En este sentido, el aprendizaje móvil se relaciona principalmente con la no dependencia de un lugar y unos tiempos, para llegar a una autonomía en los procesos y en la selección de contendidos.

Martínez \& Perales (2016), exponen: «Las personas aprenden cuando tienen la posibilidad de establecer relaciones con sentido de utilidad funcional entre los nuevos conocimientos y los que ya poseen, y cuando reciben la ayuda precisa para avanzar de forma autónoma e iniciar nuevos aprendizajes por sus propios medios» (p.140); es decir, se puede usar la tableta como dispositivo para iniciar nuevos aprendizajes por sus propios medios. 
El auge del aprendizaje universitario ubicuo. Uso de las tabletas en la apropiación del conocimiento

Blanca Inés Espinel, María luisa Sevillano García, Iván Javier Monterrosa Castro y Carolina Pascual Moscoso

En una investigación mixta comparada realizada en Colombia, Chile, Perú, Panamá, México, España, Italia y Alemania (Sevillano-García \& Vázquez-Cano, 2015), se constata que los estudiantes universitarios consideran que la tableta es buena para el trabajo académico y las relaciones sociales, con una media en la muestra del $32 \%$. El control de su pantalla táctil aparece como muy importante. Es necesario, según el 26 $\%$ de los encuestados, ejercitarse en la paciencia para adaptarse a su manejabilidad a la hora de buscar las informaciones pertinentes, pero, no solo para el aprendizaje resulta necesario este dispositivo; el desarrollo social va haciendo imprescindible su uso y, por tanto, su conocimiento en múltiples cuestiones. Se trata de una fórmula para dotar de más agilidad y eficacia a la transmisión de datos a través de las tabletas.

Sutil (2014) afirma que es imprescindible la formación para aprender el uso de la tableta en la transmisión de datos. Así, el comportamiento del estudiante tiene lugar en un entorno de práctica mediada tecnológicamente que responde a las siguientes características que propone Redecker (2008): Uso intensivo de tecnología, multitarea, capacidad de generar contenido individualmente, conectividad, inmediatez, actitud de compromiso, sociabilidad. Para ello, el uso adecuado de las tabletas, desde sus inicios, ha sido orientado hacia el consumo de contenido digital más que a la creación del mismo. Para muchos, resulta ser una forma efectiva de leer libros, artículos y revistas y recopilar información y datos, especialmente gracias a aplicaciones como Instapaper, ReaditLater y Evernote. (Aunque tampoco faltan usuarios que opinan que las tabletas invitan a distracciones que apartan de la lectura. El correo electrónico, Twitter, YouTube y Facebook son bien accesibles y con constantes avisos).

Valerio, Herrera \& Rodríguez (2014) sustentan que la tendencia a la multiplicación de dispositivos móviles constituye el nuevo paso en esta evolución. Hay que tener en cuenta que, en la creación de contenido, muchos estudiantes requieren de un teclado externo para escribir textos extensos o incluso se deciden por lápices digitales para tomar notas durante largos periodos de tiempo. Por otro lado, Manovich (2006) explica la dimensión fáctica del consumo: teléfonos móviles y redes sociales, es decir, que la imagen digital es una representación de los imaginarios sociales y formas de expresión o ideología contemporánea que se tendrá que tener en cuenta como fuente de representación del mundo moderno. 
Blanca Inés Espinel, María luisa Sevillano García, Iván Javier Monterrosa Castro y

\section{Expectativas con las tabletas}

Con respecto a la comunicación digital, Arango-Forero (2013) explica cómo se puede llegar a hacer un análisis de la información que favorezca el pensamiento complejo como una nueva forma de construcción de conocimiento por la sobreoferta de contenidos y los escenarios de redes abiertas y colaborativas; así, otorga al usuario no solo la posibilidad de seleccionar sus formas de acceder a los contenidos, sino le permite combinar naturalmente en un mismo dispositivo; es decir, que el uso de las tabletas podría ayudar a los docentes y estudiantes a seleccionar qué contenido quieren analizar.

Grande \& Cañón (2016) aseguran la necesidad de utilizar recursos útiles para estudiantes y tener habilidades complejas en tratamiento de la información que le permitan la formación de investigativa en el aula.

Fernández \& Pons, (2015) aseveran «...necesitaremos mayores competencias en herramientas para potenciar la creatividad, habilidad en la fluidez en el uso de las tecnologías de la información y de la comunicación» (p. 88). De esta forma, se aprovecha este recurso para alcanzar los objetivos que se quieren en la formación de seres pensantes.

Rodríguez \& González (2016) afirman: «...existe una cierta incoherencia en la triangulación de la información que indica la necesidad de seguir profundizando en este sentido, las tablas pueden usarse didácticamente con el propósito de formar para afrontar la actual era digital» (p. 132).

Siemens (2005) propone las competencias genéricas que se requieren para la correcta alfabetización digital de los nuevos aprendices. Con relación a este último, también Bernal (2014) analiza las posibilidades de una antropología de la comunicación en un recorrido por las categorías de la técnica, las redes sociales, el exceso de hiperconexión, el vacío y el aislamiento. Es decir, la forma cómo el hombre contemporáneo comunica su conexión con el mundo por medio de tabletas.

Cabero (2013), al respecto, dice que se «facilita el aprendizaje ubicuo y establece nuevas formas de relacionarse tanto con las personas implicadas en el proceso formativo, objeto de aprendizaje» (p. 136). Igualmente, Filippi indica que la sociedad del bienestar exige una calidad de vida que se evidencia con una formación ubicua, flexible y accesible, configurando un nuevo modelo social de formación más allá de la esencialidad, con nuevos contextos formativos innovadores, «... 
El auge del aprendizaje universitario ubicuo. Uso de las tabletas en la apropiación del conocimiento

Blanca Inés Espinel, María luisa Sevillano García, Iván Javier Monterrosa Castro y Carolina Pascual Moscoso

nuevas oportunidades, en materia educativa, la posibilidad de extender nuestro ámbito de estudio más allá de los límites físicos que nos impone la educación presencial» (Filippi, Lafuente \& Bertone, 2012, p.1).

La Unesco (2008) testifica que las tabletas están revolucionando cada una de las facetas de la vida estudiantil, considera que su uso adecuado puede contribuir al acceso universal a la educación superior, la igualdad en el ejercicio de la docencia y el aprendizaje, así como una gestión y administración de los centros más eficientes. De la misma forma, TrillosPacheco (2013) sostiene que el contexto cambiante del ámbito de la educación es uno de los más afectados, dado que la tecnología ha planteado enormes retos para su debida y pedagógica implementación en las aulas educativas.

Linne (2014) explica que, con la masificación de las tecnologías de información y comunicación, se produjeron cambios a nivel global en la mayoría de los ámbitos sociales, se evidencia que el uso intensivo de las TIC encierra una paradoja: por un lado, una mayor accesibilidad, disponibilidad e intercambio de información y por el otro, cierta dispersión, superficialidad y rapidez, que ha denominado, a partir de una categoría nativa, fast food académico.

Por su parte, Costa (2014) sostiene que las singularidades del medio móvil, integración multimedia, personalización, geolocalización y participación, son más frecuentes en las universidades, al igual que las redes sociales como canales de comunicación.

Cazaux (2008) asevera que, en el imaginario de la sociedad de la ubicuidad, en el cual las comunicaciones digitales inciden de forma categórica en el desarrollo y la evolución de los nuevos ambientes comunicativos, las figuras de emisor y receptor, así como los modelos que ayer permitían explicar el proceso comunicativo, hoy exhiben evidentes limitaciones en sus capacidades explicativas. La realidad los ha desbordado.

El desarrollo de las comunicaciones digitales móviles nos desplaza hacia un nuevo ambiente mediático: la sociedad de la ubicuidad. De acuerdo con Neil Postman (1999), detrás de toda tecnología subyace una filosofía y los principios comunicativos de la sociedad de la ubicuidad: comunicación para todos, en cualquier momento, en cualquier lugar, desplazan consigo la necesidad de producir los dispositivos de comunicaciones digitales móviles idóneos para responder a las exigencias del ambiente comunicativo que supone dicha sociedad.

Islas (2009) demuestra que el desarrollo de la web 2.0 admite ser 
Blanca Inés Espinel, María luisa Sevillano García, Iván Javier Monterrosa Castro y

considerado como un auténtico parteaguas histórico en la evolución de Internet; este impuso importantes cambios tanto en el comportamiento como en los hábitos de consumo cultural de los cibernautas.

López (2006) aporta elementos para comprender los retos que las TIC imponen a la práctica docente, desde las nuevas maneras de aprender en las llamadas «Sociedades del Conocimiento y las Redes».

Llano (2009) presenta y analiza los resultados de una investigación realizada entre 95 empresas colombianas sobre la intranet como medio de comunicación; se evidenció la alta penetración de este medio y diferentes características del papel que las áreas de comunicación han desempeñado en su desarrollo. Según esta investigación, el uso de las tabletas tiene lugar preferentemente en casa y fuera del domicilio, en los viajes, donde sustituye con frecuencia a los ordenadores portátiles. Los estudios muestran que en no pocas ocasiones se contempla la tableta como un medio apto para acostumbrar a los niños al uso de los demás dispositivos electrónicos. Además, se evidencia que, para muchos usuarios, es asociada al entretenimiento; también para ver videos utilizando el sistema W-LAN. Su poco peso constituye un elemento favorable para su transporte. El estudio se realizó sobre una muestra de 200 encuestados acerca del uso de las tabletas. Videla (2013).

\section{Metodología}

Con base a los interrogantes planteados en el estudio, se diseñó como propósito, conseguir información necesaria y suficiente sobre funciones, frecuencia y uso que le dan los estudiantes a la tableta, en los diferentes lugares objeto del estudio (Universidad de Cartagena y Fundación Universitaria Tecnológico Comfenalco). Además, conocer la opinión de los participantes: directivos y coordinadores de las diferentes instituciones educativas.

Asimismo, se procedió a dar respuesta a los interrogantes planteados en la investigación, se seleccionó por un diseño mixto desarrollado en fases complementarias. Una primera fase realizada mediante un estudio de encuesta a través de un cuestionario como metodología de estudio cuantitativo que aportaron información acerca de las ideas y percepciones de los participantes sobre el uso y frecuencia de las tabletas en su formación. En la segunda, se utilizó la técnica de grupos de discusión y 
El auge del aprendizaje universitario ubicuo. Uso de las tabletas en la apropiación del conocimiento

Blanca Inés Espinel, María luisa Sevillano García, Iván Javier Monterrosa Castro y

Carolina Pascual Moscoso

entrevistas formales a directores y coordinadores de las diferentes instituciones educativas, como metodología cualitativa con el propósito de corroborar la información obtenida mediante la encuesta.

\section{Población y muestra}

La población de directivos, coordinadores y estudiantes es en total de 213, de los cuales 100 pertenecen a la muestra de Universidad de Cartagena (UdeC) y 213 a Fundación Universitaria Tecnológico Comfenalco (Tecnológico) de los programas de administración de empresas respectivamente.

\section{Instrumentos}

Se realizó una encuesta tipo Likert, dirigida a directivos, coordinadores y estudiantes de programas de administración de empresas, donde se pudo recolectar la información con relación al uso, funciones, frecuencia del uso de la Tableta en diferentes lugares y con las variables 17.1"17.9". La fiabilidad Alfa de Cronbach es de 0,897. Se realizó también una entre vista semiestructurada.

\section{La muestra}

\begin{tabular}{lc}
\hline \multicolumn{2}{c}{ Cuestionarios recibido y tratados } \\
\hline Universidades & Número de protocolos \\
\hline Universidad de Cartagena & 100 \\
\hline $\begin{array}{l}\text { Fundación Universitaria Tecnológico de Comfenalco } \\
\text { - Cartagena }\end{array}$ & 113 \\
\hline Total & $\mathbf{2 1 3}$ \\
\hline
\end{tabular}

Para la validación de la encuesta, el tratamiento y análisis de los datos, se utilizaron los métodos descriptivos, inferencias estadísticas y análisis de contenido en textos. 


\section{Resultados y discusión}

Los Hallazgos mostrados en esta sesión son de carácter descriptivo y presentan una síntesis de las percepciones de los participantes en el estudio y con base a las variables planteadas. Tomando como bases teóricas confrontadas con las respuestas obtenidas a través de los instrumentos indicados.

Tabla 1

Usos de la tableta.

\begin{tabular}{lccc}
\hline Usos & UdeC & Tecnológico & Total \\
\hline Jugar & $12,2 \%$ & $8,5 \%$ & $20,7 \%$ \\
Buscar trabajo & $4,2 \%$ & $7,5 \%$ & $11,7 \%$ \\
Leer la prensa & $9,4 \%$ & $8,9 \%$ & $18,3 \%$ \\
Ver videos & $10,8 \%$ & $11,3 \%$ & $22,1 \%$ \\
Crear videos & $5,6 \%$ & $3,8 \%$ & $9,4 \%$ \\
Hacer grabaciones & $9,4 \%$ & $6,1 \%$ & $15,5 \%$ \\
Escuchar música & $8,9 \%$ & $9,4 \%$ & $18,3 \%$ \\
Participar en foros & $6,1 \%$ & $6,6 \%$ & $12,7 \%$ \\
Participar en redes sociales & $10,3 \%$ & $9,9 \%$ & $20,2 \%$ \\
En tareas de tipo académico & $8,9 \%$ & $7,5 \%$ & $16,4 \%$ \\
Buscar información & $9,9 \%$ & $10,8 \%$ & $20,7 \%$ \\
Organizar información & $6,1 \%$ & $8,0 \%$ & $14,1 \%$ \\
Recibir y contestar e-mail & $6,6 \%$ & $11,7 \%$ & $18,3 \%$ \\
Realizar llamadas o mensajes & $4,2 \%$ & $4,7 \%$ & $8,9 \%$ \\
\hline
\end{tabular}

Fuente: Elaboración propia.

Los datos recopilados y tratados que aparecen en la tabla 1, evidencian que la tableta en las aulas es muy poco usada. La variable (Participar en redes sociales), el mayor porcentaje lo obtuvo Universidad de Cartagena con 10,3\%, seguido de la Fundación Universitaria Tecnológico Comfenalco, con 9,9\%.

Ver videos, la encabeza el Tecnológico, con un 11,3\%; Universidad de Cartagena, con un 10,8\%. Buscar información, es liderada por Tecnológico con 10,8\%; seguida de UdeC, con 9,9\%. Recibir y contestar e-mail, obtiene una aceptación en Tecnológico con 11,7\%; seguida de Udec, con 6,6\%. Leer la prensa, la encabeza UdeC con 9,4\%; la sigue Tecnológico con 8,9\%. Escuchar música, la lidera Tecnológico con 9,4\%; seguido de UdeC, con 8,9\%. 
El auge del aprendizaje universitario ubicuo. Uso de las tabletas en la apropiación del conocimiento

Blanca Inés Espinel, María luisa Sevillano García, Iván Javier Monterrosa Castro y Carolina Pascual Moscoso

En tareas de tipo académico, UdeC con 8,9\% es la universidad en el que más alto porcentaje registra esta variable seguida de Tecnológico, con $7,5 \%$. Organizar información, la encabeza UdeC con $8,0 \%$; seguida de Tecnológico, con 6,1\%. Buscar trabajo, es liderada por Tecnológico con 7,5\%; Seguidamente, se encuentra UdeC con 4,2\%. Realizar llamadas o mensajes, la encabeza Tecnológico con 4,7\%; seguida de UdeC, con 4,2\%. Participar en foros liderada por Tecnológico con 6,6\%; UdeC, con 6,1\%. Hacer grabaciones, la encabeza UdeC 9,4\%; le sigue Tecnológico con $6,1 \%$. Crear vídeos; fue liderada por UdeC con 5,6\%; Tecnológico, con 3,8\%.

Los datos revelan que los docentes y estudiantes desconocen el uso pedagógico de las tabletas, lo cual no les permite introducirla con una mayor flexibilidad en el aula, debido a que no tienen presente que este recurso podría resultar conveniente para todos los alumnos y, en especial, para aquellos con problemas de aprendizaje.

Tal desconocimiento se pudo comprobar en los grupos de discusión, donde los docentes argumentaban que no les daban el uso a las tabletas por la falta de capacitación y consideraban que era un factor importante para la motivación.

Tabla 2

Funciones de las tabletas.

\begin{tabular}{cccc}
\hline \multirow{2}{*}{ Funciones } & \multicolumn{2}{c}{ Universidades (muestras desagregadas) } & \multirow{2}{*}{ Total } \\
\cline { 2 - 3 } & UdeC & Tecnológico & \\
\hline Entretenimiento & $26,8 \%$ & $22,5 \%$ & $49,3 \%$ \\
Expresiva & $11,3 \%$ & $11,3 \%$ & $22,6 \%$ \\
Motivadora & $8,5 \%$ & $11,3 \%$ & $19,8 \%$ \\
Informativa & $17,8 \%$ & $16,4 \%$ & $34,2 \%$ \\
Instructiva & $12,7 \%$ & $12,7 \%$ & $25,4 \%$ \\
Colaboradora & $15,0 \%$ & $12,2 \%$ & $27,2 \%$ \\
Comunicativa & $18,8 \%$ & $18,8 \%$ & $37,6 \%$ \\
Ilustrativa & $15,5 \%$ & $14,6 \%$ & $30,1 \%$ \\
Función innovadora & $26,8 \%$ & $26,3 \%$ & $53,1 \%$ \\
de la tabletas & & & \\
\hline
\end{tabular}

Elaboración propia.

Como se puede observar en la tabla 2 Sobre las funciones de la tableta. La variable Entretenimiento es utilizada, destacándose UdeC con 26,8\%, 
Blanca Inés Espinel, María luisa Sevillano García, Iván Javier Monterrosa Castro y

seguido de Tecnológico 22,5\%. Función innovadora, UdeC tiene el mayor uso con $26,8 \%$, seguida por Tecnológico con un $26,3 \%$. La variable Ilustrativa, el uso más alto lo lideró UdeC con 15,5\%, Tecnológico con $14,6 \%$. En la variable Comunicativa igualdad de valores UdeC con 18,8\%, y Tecnológico 18,8\%. La función Informativa, UdeC es la de mayor porcentaje 17,8\%, y Tecnológico con 16,4\%. En la función Colaboradora. UdeC el mayor con $15,0 \%$, y Tecnológico con $12,2 \%$. En la variable Expresiva, UdeC con un 11,3\%, y Tecnológico con 11,3\%. Por último la variable Motivadora, registra porcentajes de uso Tecnológico con $11,3 \%$, y UdeC con $8,5 \%$.

Se puede comprobar que las tabletas son muy poco utilizadas como un dispositivo pedagógico en la educación superior. Por lo que se desconoce que ésta permite introducir una mayor flexibilidad en el aula y favorecer el aprendizaje personalizado. Además, se desperdicia la oportunidad de valorar un instrumento que contribuye al cambio y a la modificación de metodologías en la práctica docente. Además, no se le da el valor que esta presenta de forma inmediata de la información actualizada que se requiere y que es una fuente inagotable de información multimedia e interactiva, y que facilita la relación de los contenidos curriculares con el contexto.

En relación con la utilización de la Tableta en la búsqueda de información académica se obtuvieron los siguientes resultados, que presentamos en la tabla. 3.

Tabla 3

Utilización de la Tableta en la búsqueda de información académica.

\begin{tabular}{cccccc}
\hline \multirow{2}{*}{ Universidades } & \multicolumn{5}{c}{ Opciones } \\
\cline { 2 - 6 } & Nada & Casi nada & De vez en cuando & A menudo & Siempre \\
\hline UdeC & $35,3 \%$ & $3,4 \%$ & $2,5 \%$ & $3,4 \%$ & $2,5 \%$ \\
Tecnológico & $21,8 \%$ & $5,0 \%$ & $10,1 \%$ & $6,7 \%$ & $9,2 \%$ \\
\hline Total & $57,1 \%$ & $8,4 \%$ & $12,6 \%$ & $10,1 \%$ & $11,8 \%$ \\
\hline
\end{tabular}

Elaboración propia.

Según datos de la tabla 3, los estudiantes universitarios no la emplean en la búsqueda de información académica, Nada, equivale a un promedio $57,1 \%$, Mientras que Siempre $11.8 \%$. Se deduce que existe desconocimiento sobre su uso con fines académicos, ya que puede captar mejor 
la atención de los estudiantes, favorece la motivación en la comprensión de los tópicos y se incrementa la memoria visual. Esto les lleva a desconocer que la Tableta mejora determinadas competencias de los estudiantes, sobre todo las referidas a la búsqueda de información o el manejo de las TIC y que es uno de los últimos recursos tecnológicos que invitan a la innovación pedagógica y además se puede considerar que es una herramienta que favorece el desarrollo de la autonomía personal y que con este dispositivo se consigue reforzar la creatividad de los educandos.

La reflexión anterior comprueba una vez más el poco uso que se le da a la Tableta como recurso didáctico, reconociendo que hay un desconocimiento sobre uso y que permite el acceso universal a la educación. Beneficia la igualdad en el ejercicio de la docencia y el aprendizaje, así como una mejor sencilla y eficaz gestión. Además, puede servir de introducirla en el aula al en el aprendizaje de los estudiantes. A través de los Tableta se puede favorecer la metacognición y autorregulación del aprendizaje.

Asimismo, se puede trabajar con ayuda de la tableta la comprensión de los textos, observar, criticar, analizar, encontrar semejanzas y diferencias, las competencias digitales, la creatividad, la motivación o el estudio de forma autónoma. 
El auge del aprendizaje universitario ubicuo. Uso de las tabletas en la apropiación del

conocimiento

Blanca Inés Espinel, María luisa Sevillano García, Iván Javier Monterrosa Castro y

Carolina Pascual Moscoso

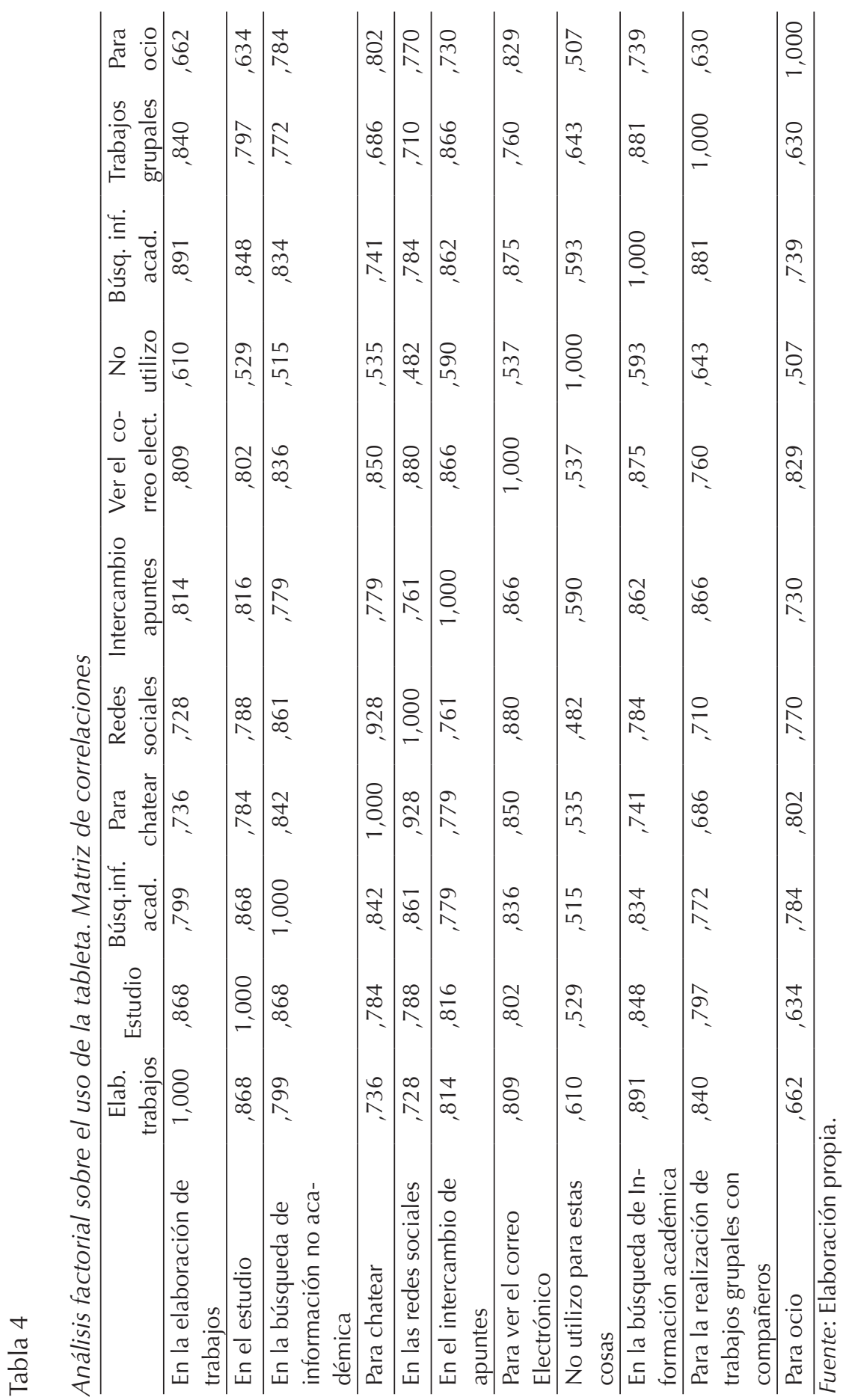

Educatio Siglo XXI, Vol. 37 n $2 \cdot 2019$, pp. 183-204 
El auge del aprendizaje universitario ubicuo. Uso de las tabletas en la apropiación del conocimiento

Blanca Inés Espinel, María luisa Sevillano García, Iván Javier Monterrosa Castro y Carolina Pascual Moscoso

El análisis factorial realizado, muestra los resultados que aparecen en la tabla 4. El mayor porcentaje fue el factor búsqueda de Información académica 0,891; seguido de En el estudio, con 0,868. El factor para la realización de trabajos grupales con compañeros, 0,840, En el intercambio de apuntes, 0,814. Para Para ver el correo Electrónico 0,809.

Se pudo comprobar a través de la encuesta que la gran mayoría de los estudiantes utilizan las tabletas sin ninguna orientación pedagógica solo como gestión de redes sociales (Twitter, FaceBook, Linkedln) y todo tipo de foros, realizan videoconferencias (especialmente fácil en dispositivos iPad con la aplicación FaceTime), envío de mensajes SMS y Ilamadas telefónicas a través de Internet (voz IP) sin costos añadidos

Según los resultados de la tabla anterior, la tableta es una herramienta que hace experimentar la innovación, integrar, evaluar y producir cambios esenciales en la construcción del propio aprendizaje en el alumnado y la de establecer nuevos roles en la relación profesor-alumno. La forma en que los estudiantes adquieren y construyen conocimientos producirá efectos mucho más permanentes y efectivos tanto en el proceso individual como en la capacidad de la sociedad para adaptarse a un mundo cambiante.

En este sentido, las respuestas obtenidas por los colaboradores en los diferentes ítems de la encuesta evidencian quizá sea debido a la poca o ninguna formación que tiene el profesorado sobre el uso pedagógico para así, cambiar sus prácticas pedagógicas necesarias e imprescindible para su implantación en el aula.

Profundizando más allá de este análisis general y ubicando las distintas dimensiones que han sido objeto de análisis en esta investigación, se ve que se desconoce pero la tableta como dispositivo tecnológico que permite la ubicuidad que conforma la acción actualmente de los estudiantes. Al ser un dispositivo relativamente nuevo es visto por parte de los jóvenes como atractivo, por ello su uso querido e intensivo; por otro lado, los mayores lo ven como algo que puede distraer. 


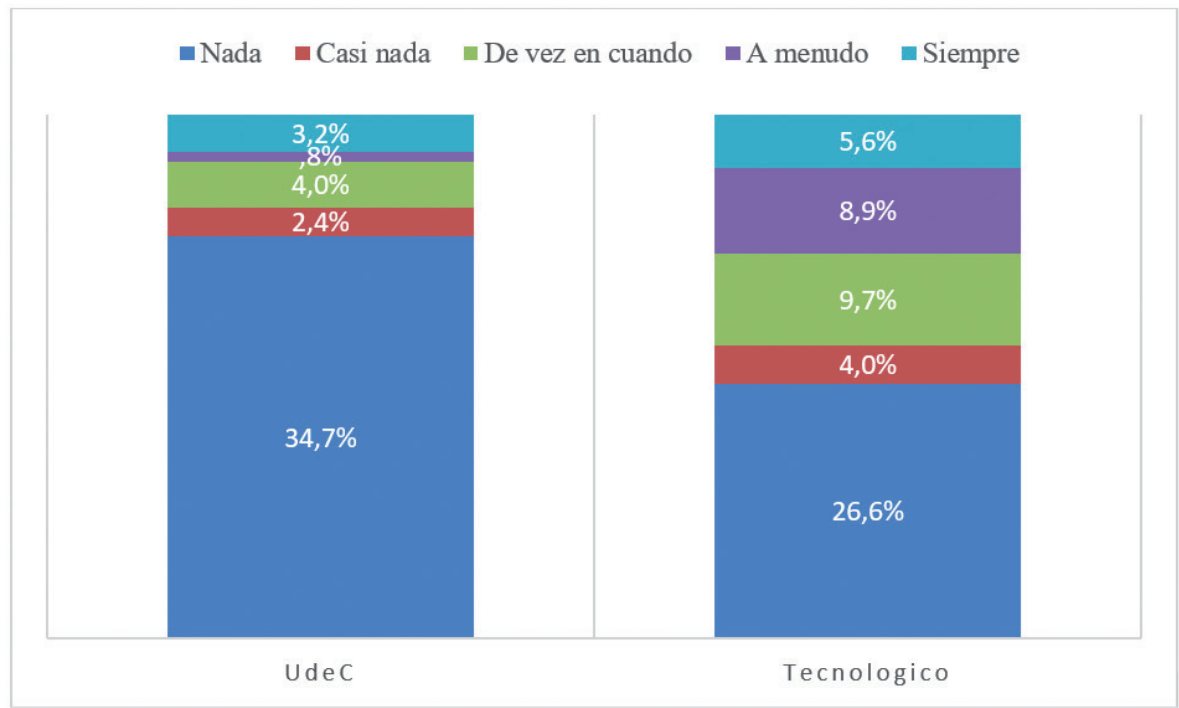

Figura. 1. Cuadro Comparativo empleo de la tableta con fines didácticos Fuente: Elaboración propia.

El uso de las tabletas, con fines educativos el porcentaje de la categoría nada es similar entre las dos universidades. Se puede evidenciar que en la primera Universidad el porcentaje es del $34,7 \%$ y en la otra universidad el porcentaje es del $26,6 \%$. Se constata que en cuestiones de uso de nuevas tecnologías en la enseñanza y el aprendizaje hay algo más que la mera distribución de artefactos. Hay acciones como la formación, la investigación, el asesoramiento que pone en valor el conocimiento inherente a la tecnología transferida ya señalado en el marco teórico. Además, los profesores perciben que tienen en sus aulas sujetos atravesados por la cultura digital, jóvenes de la generación multimedia, nativos digitales, adolescentes del siglo XXI.

\section{Conclusiones}

El análisis general de los resultados de esta investigación muestra que la tableta ha experimentado tal grado de uso que ya no puede pensarse un presente ni futuro sin ella. Se ha verificado su utilidad y valor y sus múltiples prestaciones.

Se constata que es necesario el aprovechamiento de los recursos pe- 
El auge del aprendizaje universitario ubicuo. Uso de las tabletas en la apropiación del conocimiento

Blanca Inés Espinel, María luisa Sevillano García, Iván Javier Monterrosa Castro y Carolina Pascual Moscoso

dagógicos dispuestos en internet, así como la necesidad de garantizar la mayor promoción y generación de profesores eficaces en los escenarios de enseñanza superior a nivel de apropiación tecnológica con las tabletas.

Según este estudio, los estudiantes universitarios usan las tabletas, pero dada su formación y educación no se apropian de sus posibilidades productivas más allá de los usos básicos o recreativos. La apropiación tecnológica es un fenómeno individual pero también colectivo que incluye factores políticos, económicos y culturales que deben ser analizados en su conjunto.

Saben que la imagen de un profesor transmisor de la información, protagonista central de los intercambios entre sus alumnos y guardián del currículum ha entrado en crisis en un mundo conectado por pantallas. Son conscientes que se les demanda una enseñanza renovada, ajustada a las características de estos nuevos alumnos

Con este estudio, se focalizó el aprovechamiento de la tableta en espacios tradicionales y nuevos; de esta manera, se convierte en un elemento primordial para el desarrollo del currículo, pero que aún no ha sido valorado por los estudiantes universitarios para su formación académica (bien sea por las desigualdades en el acceso a este dispositivo tecnológico), ni por parte de las familias, ni por las instituciones universitarias.

De la misma manera, es poco el uso que se le da a la tableta en el ámbito universitario, teniendo en cuenta que puede servir para el desarrollo competencias generales:

- La actual Sociedad de la Información y el Conocimiento en la Educación Superior demandan las siguientes competencias: Tratamiento de la información, 29\%; Lengua Extranjera, 26\%; Análisis y Síntesis, 20,8\%; Mecánicas, 17\%; Digital, 13,2\% y Comunicativa, $3,8 \%$.

- Las tabletas en el aprendizaje pueden contribuir significativamente al desarrollo de las competencias, lo que implica que el uso adecuado, justificado y aplicado de estos dispositivos es percibido por los estudiantes como instrumentos y recursos con gran potencial en su carrera profesional y académica.

- La tableta es uno de los instrumentos y recursos tecnológicos con mayor potencialidad, aunque su integración didáctica en el Espacio Europeo en la Educación Superior es todavía incipiente con 
Blanca Inés Espinel, María luisa Sevillano García, Iván Javier Monterrosa Castro y

aplicaciones aplicadas muy limitadas para el desarrollo competencial de las asignaturas universitarias.

- El uso que realiza el estudiante es de tipo personal, como se ha reseñado. De manera creciente, los sujetos se ven inmersos dentro de un nuevo hábitat de aprendizaje en el que sus mundos vitales se configuran como medios y recursos potenciales para el aprendizaje, en el que su experiencia es consignada individualmente en relación con las acciones que es capaz de desarrollar, ya sea en su vida cotidiana o formando parte de planes de estudios.

- Otra tendencia importante, que requiere respuestas pedagógicas, es el predominio de los llamados «contextos generados por el usuario».

- En la medida en que los usuarios participan activamente en la elaboración de sus propias formas de generación de contenidos, los contextos de aprendizaje también se circunscriben al ámbito de lo individual. Esto motiva el surgimiento de nuevas relaciones entre el contexto y la realización de prácticas de comunicación móviles enteramente gestionadas por el usuario.

- En el caso de la educación, las tabletas permiten una relación novedosa del estudiante con el espacio (el mundo exterior) y con el lugar (el entorno socio-cultural próximo). Igualmente, las tabletas alteran la secuencia de comunicación tradicional entre los agentes educativos. Los estudiantes son ahora productores de contenido y forman parte de una explosión de actividad en el área del contenido generado por otros usuarios, incluidos los docentes.

Comunidad científica universal, instituciones, docentes, familias, estudiantes, están llamados a redescubrir en estos maravillosos inventos del ingenio humano, aliados colaboradores de la gran tarea de comunicar y enseñar más fácilmente y mejor en favor de una sociedad nueva.

\section{Referencias}

Alonso, A. S.M., Domínguez, J. M., y Orellana, E. R. (2016) Recepción y asimilación de las tecnologías en centros escolares: El proyecto "El rincón del ratón". Educación XX1, 19(2) 337-358. doi10.5944/educXX1.13944

Arango-Forero, G. (2013). Comunicación digital: una propuesta de análisis desde el pensamiento complejo. Palabra Clave 16 (3), 673-697. 
El auge del aprendizaje universitario ubicuo. Uso de las tabletas en la apropiación del conocimiento

Blanca Inés Espinel, María luisa Sevillano García, Iván Javier Monterrosa Castro y

Carolina Pascual Moscoso

Bernal, P. (2014). ¿Es aún posible la comunicación en medio del imperio de la incomunicación? Palabra Clave 17(2), 294-319.

Berrio-Zapata, C. y Rojas-Hernández, H. (2014). La brecha digital universitaria: La apropiación de las TIC en estudiantes de Educación Superior en Bogotá (Colombia) Comunicar: Revista Científica de Comunicación y Educación, 22(43), 133-142. Doi http://dx.doi.org/10.3916/C43-2014-13.

Cabero Almenara, J. (2013). El aprendizaje autorregulado como marco teórico para la aplicación educativa de las comunidades virtuales y los entornos personales de aprendizaje. Teoría de la Educación: Educación y Cultura en la Sociedad de la Información 14(2), 133-156.

Cazaux, D. (2008). La Comunicación Pública de la ciencia y la Tecnología en la Sociedad del Conocimiento. Razón y Palabra 13(65), 2-12.

Chiecher, Costa, A.C. y Lorenzati Blengino, K.P. (2017) Estudiantes y tecnologías. Una visión desde la "lente" de docentes universitarios. RIED. Revista Iberoamericana de Educación a Distancia 20 (1), 261-282. Doi: http.// dx.doi.org/10.5944/. ried.20.1.16334.

Concilio Vaticano II. Constituciones, Decretos, Declaraciones. Decreto Inter Mirifica Madrid: BAC.

Cossío Gutiérrez, E. F., \& Hernández Rojas, G. (2016). Las teorías implícitas de enseñanza y aprendizaje de profesores de primaria y sus prácticas docentes. Revista mexicana de investigación educativa, 21(71), 1135-1164.

Costa-Sánchez, C. (2014). Las singularidades del medio móvil: integración multimedia, personalización, geolocalización y participación. Estudio de su presencia en las apps de la prensa española. Palabra Clave 17 (3), 672-694.

Durán Cuartero, M., Gutiérrez Porlán, I., \& Prendes Espinosa, M. P. (2016). Certificación de la competencia TIC del profesorado universitario: Diseño y validación de un instrumento. Revista mexicana de investigación educativa, 21(69), 527-556.

Fernández, M. À. P. I., \& Pons, E. S. O. (2015). ¿Pueden las TIC mejorar los resultados académicos? Diseños formativos y didácticos con soporte TIC que mejoran los aprendizajes: el caso de los contenidos digitales de ortografía de Digital-Text. Educatio Siglo XXI, 33(3), 85-102.

Filippi, J. L., Lafuente, G. J., \& Bertone, R. A. (2012). Diseño de un ambiente de aprendizaje colaborativo. Revista Iberoamericana de Educación a Distancia, 10 (2), 155-166.

Grande-De-Prado, M., Cañón-Rodríguez, R., \& Cantón-mayo, I. (2016). Competencia digital y tratamiento de la información en futuros maestros de Primaria. Educatio Siglo XXI, 34(3), 101-118.

Hagen, L., Renatus, R. \& Schenk, S. (2013). Mediale Ubiquität als Faktor jugendlicher. MERZ, 6, 53-71.

Hung, E. S., Díaz-Granados, F. I., Molinares, D. J., Barreto, C. R., Ballesteros, B., Vergara, E, y Ordoñez, M. (2015) Fortalecimiento pedagógico en las universidades en Colombia a través de las TIC. Caso región Caribe. Educación XX1, 18(2), 277-304. doi10.5944/educXX1.14019

Ibáñez, J. S. (2004). Innovación docente y uso de las TIC en la enseñanza universitaria. RUSC. Universities and Knowledge Society Journal, 1(1), 3. 
El auge del aprendizaje universitario ubicuo. Uso de las tabletas en la apropiación del conocimiento

Blanca Inés Espinel, María luisa Sevillano García, Iván Javier Monterrosa Castro y Carolina Pascual Moscoso

Islas, O. (2009). El prosumidor. El acto comunicativo de la sociedad de la ubicuidad. Palabra Clave, 1 (11), 29-40.

Katja, F. (2012). Mobiles Lernen in der Schule. En: Laufer, J. und Röllecke, R, \& Chancen: Digitaler Medien für Kinder und Jugendliche. München: Kopaed.

Linne, J. (2014). Ahora hago fast food académico. Los estudiantes de la Universidad de Buenos Aires en tiempos de Internet. Palabra Clave 17(3), 695-716.

Llano Aristizábal, S. (2006). La intranet en organizaciones colombianas. Palabra Clave 9(1), 99-110.

López Jiménez, D (2006). El conocimiento y la comunicación: dos pilares fundamentales de la organización de la sociedad de la información. Palabra Clave 9(2), 91-109.

Manovich, L. (2006). El lenguaje de los nuevos medios de comunicación: la imagen en la era digital. Buenos Aires: Paidós.

Martínez-Garrido, C., \& Murillo, F. J. (2016). Investigación iberoamericana sobre enseñanza eficaz. Revista mexicana de investigación educativa, 21(69), 471-499.

Martínez-Villar, A., Gutiérrez-Pérez, J., \& Perales-Palacios, F. J. (2016). Evaluando la formación virtual en sensibilización ambiental para sectores profesionales. Educatio Siglo XXI, 34(3), 137-160.

Mas-Torelló, O. \& Olmos-Rueda, P. (2016). El profesor universitario en el Espacio Europeo de Educación Superior: la autopercepción de sus competencias docentes actuales y orientaciones para su formación pedagógica. Revista mexicana de investigación educativa, 21(69), 437-470.

Postman, N. (1999). El fin de la educación. Barcelona: Octaedro.

Rodríguez-Izquierdo, R. M., \& González-Pérez, A. (2016). Uso pedagógico de la pizarra digital interactiva en la enseñanza y el aprendizaje. Un estudio de caso. Educatio Siglo XXI, 34(3), 119-136.

Said-Hung, E, Iriarte Díaz-Granados, F. Jaba Molinares, D.; Ricardo Barreto, C, Ballesteros, B, Vergara, E, y Ordoñez, M. (2015) Fortalecimiento pedagógico en las universidades en Colombia a través de las TIC. Caso región Caribe. Educación XX1, 18 (2) 277-304, doi10.5944/educXX1.14019

San Martín, A., Martín, J. \& Ramírez, E. (2016) Recepción y asimilación de las tecnologías en centros escolares: El proyecto "el rincón del ratón ". Educación XX1, 19(2) 337-358, doi10.5944/educXX1.1394

San Martín, O. (2014, 24 de abril). Los aspirantes a profesores serán evaluados de idiomas y tecnologías. Diario El Mundo, p. 21.

Sevillano-García, M.L. \& Vázquez-Cano, E. (2015). Modelos de investigación en contextos ubicuos y móviles en Educación Superior. Madrid: McGraw-Hill.

- (2015). The Impact of Digital Mobile Devices in Higher Education. Educational Tecnology \&Society 18 (1), 106-118.

Siemens. G. (2005). Conectivismo: Una teoría de la enseñanza para la era digital. International Journal of Instructional Technology and Distance Learning 2 (10), p.1.

Sutil, T. (2014, 8 de marzo). Las elecciones europeas Ilaman al voto. La Opinión de Zamora, p.1.

Unesco (2005). Hacia las sociedades del conocimiento. Mayenne: Ediciones Unesco.

Valerio, G., Herrera-Murillo, D. J., \& Rodríguez-Martínez, M.C. (2014). Asociación entre 
El auge del aprendizaje universitario ubicuo. Uso de las tabletas en la apropiación del conocimiento

Blanca Inés Espinel, María luisa Sevillano García, Iván Javier Monterrosa Castro y

Carolina Pascual Moscoso

El momento de publicación en las redes sociales y el engagement: estudio de las universidades mexicanas. Palabra Clave 17 (3), 749-772.

Videla-Rodríguez, J. J. \& Piñeiro-Otero, T. (2013). La radio móvil en España. Tendencias actuales en las apps para dispositivos móviles. Palabra Clave 16 (1), 129-153.

Torres, M. P. A., \& Ponce, F. C. (2015). Los libros de texto digitales en México: un apoyo al trabajo didáctico en las aulas de educación básica. Educatio Siglo XXI, 33(3), 103122.

Trillos-Pacheco, J. J. (2013). La lectura hipermedial y su incidencia en la comprensión lectora en estudiantes universitarios. Palabra Clave 16 (3), 944-992. 